Hieraus scheint mir unzweifelhaft bervorzugehen, dass Estlander's theoretische Begründung seiner Operation durchaus unzutreffend ist; es bleibt also nur die Art der Ausführung, für welche Sie Estlander's Priorität in Anspruch nehmen. Das muss ich aber, unter Hinweis auf den Artikel in meinem Buche: "Fünf Jahre im Augustahospital" bestreiten, da ich in demselben bereits 1876, im Anschluss an mehrere operirte Fälle, eine eingehende Darlegung der Verhältnisse bei alten Empyemen gegeben und dieselbe Operation, behufs Verkleinerung des Thorax, vorgeschlagen habe. Der ganze Unterschied liegt höchstens in der grösseren Zahl der Rippenstücke, welche Estlander fortnimmt, die er aber selber als wahrscheinlich bedeutungslos hinstellt, und in der grösseren Zahl der Rippen, die er resecirt, nämlich $3-7$, während ich nur von $2-4$ spreche. Eine nunmehr sehr ausgedehnte Erfahrung hat mich gelehrt, dass diejenigen Fälle, in welchen man mit der Resection von vier Rippen nicht auskommt, wahrscheinlich immer unheilbar sind, nämlich alle diejenigen, in welchen die Lunge, ohne jede Adbäsion, zu einem Klumpen geballt, der Lungenwurzel anhängt.

Hiernach muss ich noch einmal mit aller Schärfe den Anspruch erheben, der erste gewesen zu sein, welcher für sämmtliche Formen von Empyem, auch für die veralteten Brustfisteln mit Lungenschrumpfung, rationelle Behandlungsgrundsätze aufgestellt und wissenschaftlich begrürdet hat. Die Berechtigung der Bezeichnung der Estlander'schen Operation kann ich also nicht anerkennen; höchstens kann ich Estlander das Verdienst zuschreiben, ohne Kenntniss meiner Arbeit auf den gleichen Gedanken gekommen zu sein; wie ich, und für die Verbreitung dieses Gedankęns, durch Publication in einer viel gelesenen Zeitschrift, besser gesorgt zu haben.

So sehr es Ihnen Ehre macht, für die litterarischen Arbeiten Ihres Vorgängers, dessen Verdienste auf anderen Gebieten der Chirurgie ich vollauf anerkenne, eingetreten zn sein, so darf ich mich dennoch wohl der Hoffnung hingeben, dass eine genaue Vergleichung unserer Arbeiten Sie von meinen Prioritätsrechten überzeugen wird. Ich zweifle nicht daran, dass der ver-

\section{Offener Brief an Herrn Professor Dr. Saltzmann in Helsingfors.}

Sehr verehrter Herr College!

Als ich vor einigen Jahren die Ehre hatte, Sie als Gast auf meiner Abtheilung im Augustahospital begrüssen zu dürfen, geriethen wir in eine Discussion über die sogenannte Estlander'sche Methode der Empyemoperation, für welche ich die Priorităt in Anspruch nahm. Dies Gesprä̀ch verlief indessen ohne Resultat,'weil Sie den Aufsatz, auf welchen ich meinen Anspruch gründete, nicht kannten, und ich von der Estlander'schen Arbeit nur im Auszuge Kenntniss genommen hatte. Inzwischen hat unsere Discussion mit besserer Vorbereitung ihre Fortsetzung gefunden, da Sie einige Bemerkungen in meinem Vortrage: „Ueber die Grundsätze der Behandlung von Eiterungen in starrwandigen Höhlen, mit besonderer Berücksichtigung des Empyems der Pleura" (diese Wochenschrift No. 10-13) in der Nummer vom 18. April dieses Jahres einer Kritik unterzogen haben. Wenn meine Antwort erst so spät erscheiut, so erklärt sich das aus den Schwierigkeiten, welche ich bei der Beschaffung der Revue mensuelle Tom. III behufs erneuter Durchsicht der Estlander'schen Arbeit gefunden habe.

In Ihrer Erwiderung begrüsse ich es zunächst mit Freuden, dass Sie die Bezeichnung der Operation eines Empyems mit mebrfachen Rippenresectionen, als Estlander'scher Operation, gleich mir verwerfen. In der That kann eine solche Bezeichnung nur der Unkenntniss der beiden Estlanderschen Artikel (a. a. O.) ihren Ursprung verdanken, und ist $\mathrm{zu}$ hoffen, dass dieselbe in Zukunft fallen gelassen werde.

Thre Kritik wendet sich indessen mit besonderem Nachdruck gegen meine Bemerkung, Estlander habe die Operation erst dann auszuführen empfohlen, wenn die Eiterung lange genug bestanden habe, um Verdickungen der Pleura zu erzeugen; denn erst diese machten die Operation leicht und gefahrlos. Diese Ausfübrungen, füge ich später hinzu, haben sich als keineswegs zutreffend erwiesen. Sie erklären diese meine Auffassung als irrthümlich, weil Estlander's ,Thoracoplasti“ mit der Eröfnung einer eiternden Pleurahöhle durch partielle Resection einer Rippe garnichts $\mathrm{zu}$ schaffen habe, sondern ausschliesslich eine Verringerung des Thoraxumfanges und Anlegung der Pleura costalis an eine geschrumpfte Lunge bei lange bestehender Brustfistel oder Empyema necessitatis bezwecke. Die Unterscheidung, welche Sie hier machen, scheint mir ein wenig künstlich; denn wenn auch zugegeben werden muss, dass es ein grosser Unterschied ist, ob man eine Rippe resecirt, nur um dem Eiter dauernd freien Abfluss zu verschaffen, oder in der ausgesprochenen Absicht den Thorax zu verkleinern, so ist doch das Ziel beider Operationen, wenigstens bei alten Empyemen, dasselbe, nămlich eine baldige Anlegung des Thorax an die Lunge zu bewirken. Dass Estlander dies Ziel besser zu erreichen glaubte nach langer, als nach kurz dauernder Eiterung, geht aus folgendem Satz seiner Arbeit unzweifelhaft hervor: "S'il est important, que les empyèmes qu'on traite par la résection des côtes soient anciens, parce que la rétraction de la plèvre épaissie est alors plus forte, cette condition est encore plus importante quant à l'opération méme, car on peut dire qu'en général la possibilité d'un heureux résultat dẻpend du changement de la plèvre." An einer früheren Stelle sagt er geradezu: "Plus la maladie est ancienne, mieux cela vaut". Estlander legt also allen Nachdruck auf die Verdickung der Pleura und deren nachtrăgliche Schrumpfung und lässt die Wiederausdehnung der Lunge, auf welche ich gauz allein für die Heilung älterer Empyeme Gewicht lege, gänzlich aus dem Bereich der Erörterung. Dieser durchaus unzutreffenden Theorie zur Liebe lässt er also die Eiterung länger bestehen, um nur ja eine narbig verdickte Pleura zu bekommen. Eine solche Auffassung ist durchaus von der Hand zu weisen, und ist dies auch bereits von Th. Kölliker, dem Referenten über die Estlander'sche Arbeit (Centralbl. f. Chirurgie 1879), geschehen. Ich füge hinzu, dass sie im diametralen Gegensatz zu den jetzt herrschenden Anschauungen steht, welche darauf hinauslaufen, dass es für den Patienten um so vortheilhafter ist, je frühzeitiger die Operation, ohne oder mit der Absicht den Thorax zu verkleinern, unternommen wird. storbene Estlander selber, nach Kenntnissnahme meines Artikels, diesen Anspruch anerkannt haben würde.

Mit collegialem Gruss Ihr ergebenster

Berlin, den 8. Juni 1889. 